\title{
FRECUENCIA DE CÁNCER EN PACIENTES CON ECOGRAFÍA MAMARIA BI-RADS 4A
}

\author{
José Ismael Guío MD*, Óscar A. García MD**, Mayury Liliana Buitrago MD***
}

\section{Resumen}

Las imágenes son fundamentales en la evaluación de la patología mamaria. El Colegio Americano de Radiología estandarizó los reportes con el Breast Imaging Reporting and Data System (BI-RADS), que permite predecir según las características morfológicas la probabilidad de malignidad y la conducta a seguir. En la cuarta edición de 2003 se amplió para incluir el primer lexicón de ecografía. Los BI-RADS 4 y 5 corresponden a lesiones sospechosas de malignidad y en la 4 fue necesario crear tres subgrupos, de los cuales el $4 \mathrm{~A}$ son lesiones con posibilidad baja de cáncer (entre $2 \%$ y $10 \%$ ). Como en la primera publicación presentamos los resultados del valor predictivo positivo del reporte BI-RADS 4A mamográfico, el objetivo del presente estudio es determinar la tasa de malignidad en BI-RADS 4A ecográfico en los hospitales de San José e Infantil Universitario de San José de Bogotá DC, Colombia, incluyendo casos de BI-RADS 4A mamográfico para contrastar con el estudio inicial. Cuatro de 72 pacientes con ecografía mamaria BI-RADS 4A fueron diagnosticadas con cáncer de mama (VPP del 5.5\%), todos en nódulos sólidos. Algunos pudieron catalogarse como BI-RADS 3 por los radiólogos.

Palabras clave: ecografía mamaria, mamografía, BI-RADS 4A, cáncer de mama.

\section{RATE OF BREAST CANCER DIAGNOSIS IN PATIENTS CATEGORIZED AS BI-RADS 4A ON SCREENING ULTRASOUND}

\section{Abstract}

\begin{abstract}
Imaging is essential for breast pathology evaluation. The American College of Radiology provides a standardized classification system the Breast Imaging-Reporting and Data System (BI-RADS), allowing prediction of malignancy probability based on appearance and providing guidelines to be followed. The 2003 fourth edition was extended to include the first ultrasound lexicon. BI-RADS 4 and 5 categories are lesions with suspicious changes of malignancy, and category 4 was divided into three sub groups, $4 \mathrm{~A}$ lesions have low possibility of cancer (between $2 \%$ and $10 \%$ ). As our first publication presented the results of the positive predictive value of BI-RADS 4A mammographic reports, the objective of the present study is to determine the malignancy rate in BI-RADS 4A ultrasound reports at San José and Infantil Universitario de San José hospitals in Bogotá DC, Colombia, including BI-RADS 4A mammographic exam cases to compare with the initial study. Of the 72 BI-RADS 4a lesions on ultrasound screening, four were malignant (VPP 5.5\%) all detected as solid nodules. Some could have been categorized as BI-RADS 3 by radiologists.
\end{abstract}

Key words: breast ultrasound, mammogram, BI-RADS 4A, breast cancer

Fecha recibido: abril 28 de 2015 - Fecha aceptado: mayo 7 de 2015

* Ginecólogo y Obstetra. Especialista en Mastología. Instructor Asistente. Coordinador del Programa de Mastología. Fundación Universitaria de Ciencias de la Salud. Hospitales de San José e Infantil Universitario de San José. Bogotá DC, Colombia.
** Cirujano General. Especialista en cirugía de seno y tumores de tejidos blandos. Instructor Asistente. Coordinador del programa de cirugía de seno y tumores de tejidos blandos. Fundación Universitaria de Ciencias de la Salud. Hospitales de San José e Infantil Universitario de San José. Bogotá DC, Colombia.

*** Residente IV de Ginecología y Obstetricia. Fundación Universitaria de Ciencias de la Salud. Bogotá DC, Colombia. 


\section{Introducción}

Las imágenes han jugado un papel importante en la evaluación complementaria de la mama. ${ }^{1}$ Para mejorar la estandarización del reporte imaginológico, el American College of Radiology propuso el Breast Imaging Reporting and Data System (BI-RADS), herramienta que define criterios unificados para reportar los hallazgos encontrados en la mamografía. ${ }^{2,3}$ Este sistema permite predecir de acuerdo con las características morfológicas de la lesión la probabilidad de malignidad y según ello la conducta a seguir. ${ }^{4}$ Dada la importancia creciente y el amplio uso de la ecografía mamaria, el Colegio amplió el BI-RADS para incluir el primer lexicón de ecografía en su cuarta edición publicada en el año 2003. ${ }^{5}$

Las categorías BI-RADS 4 y 5 corresponden a lesiones sospechosas de malignidad. La categoría 4 incluye lesiones con un espectro muy amplio de probabilidad de malignidad, por lo cual fueron creados tres subgrupos, dentro de los cuales, la subcategoría 4A corresponde a lesiones con una posibilidad baja de cáncer (entre $2 \%$ y $10 \%)^{5}$

En una publicación previa hemos presentado los resultados de un estudio en el cual se evaluó el valor predictivo positivo del reporte de BI-RADS 4A mamográfico. ${ }^{6}$ El objetivo del presente estudio es determinar la tasa de malignidad en casos de BI-RADS 4A ecográfico en una cohorte de pacientes atendidas en la consulta de mastología de los hospitales de San José e Infantil Universitario de San José de Bogotá DC, Colombia, también se incluyeron casos de BI-RADS 4A mamográfico con el objeto de contrastar estos últimos resultados con los del estudio inicial. Cuatro de 72 pacientes con ecografía mamaria BI-RADS 4A fueron diagnosticadas con cáncer de mama (VPP del 5.5\%), todos en nódulos sólidos. Muchos de estos estudios pudieron ser catalogados como BI-RADS 3 por parte de los radiólogos.

\section{Materiales y métodos}

Se trata de un estudio observacional descriptivo tipo serie de casos con aprobación por el comité de in- vestigaciones y ética de la facultad de medicina de la Fundación Universitaria de Ciencias de la Salud, Hospital de San José e Infantil Universitario de San José.

Se incluyeron pacientes que asistieron a la consulta de seno entre noviembre 2010 y marzo 2012. Los criterios de inclusión fueron: ser mujer, reporte de BI-RADS 4A en la mamografía, en la ecografía mamaria o en ambas, realizadas máximo doce meses antes de la consulta, sin importar el centro radiológico del cual provinieran.

Todas las pacientes fueron valoradas por un mastólogo quien definió la conducta y el seguimiento. No se evaluó la concordancia entre los observadores iniciales del reporte imaginológico y la lectura realizada en la consulta de seno. Se indagó por edad, menarquía, menopausia, nacimiento de hijos, uso de terapia de reemplazo hormonal y antecedente personal o familiar de cáncer de mama hasta el segundo grado de consanguinidad. Se incluyeron variables como motivo de consulta, indicación del procedimiento y localización por cuadrantes dentro de la glándula mamaria.

Las pacientes fueron sometidas a biopsia, la cual se llevó a cabo mediante guía ecográfica, por estereotaxia cuando la lesión no era palpable (para ello se remitieron a otras entidades para su realización), o mediante biopsia abierta previa marcación con arpón, guiado por ecografía mamaria o mamografía. La mayoría de estudios histopatológicos se analizaron en el Hospital de San José e Infantil Universitario de San José. Se hizo seguimiento a las pacientes hasta obtener el resultado definitivo de patología.

Se estudió la frecuencia de lesiones malignas de acuerdo con el hallazgo imaginológico, el tipo de biopsia utilizada y el número de ellas que se necesitaron hasta llegar a un diagnóstico histopatológico concreto. Cuando se documentó malignidad, se determinó el tipo histológico, el estado de receptores hormonales (estrógeno, progesterona), la sobreexpresión del oncogén HER-2 y el estadio clínico.

La base de datos se construyó en Microsoft Excel 2007; para el análisis de datos se utilizó el programa Stata 12. Teniendo en cuenta la prueba diagnóstica (mamografía, ecografía mamaria o ambas) se descri- 
ben las variables cuantitativas mediante promedios y desviación estándar, las variables categóricas se presentan como variables absolutas y relativas.

\section{Resultados}

Se identificaron 121 pacientes que asistieron a la consulta de mastología en el Hospital de San José e Infantil Universitario de San José entre noviembre 2010 y marzo 2012, con hallazgo imaginológico de BI-RADS 4A por mamografía, ecografía mamaria o ambas. Dieciseis pacientes no concluyeron el estudio debido a limitaciones con los trámites administrativos. Se identificaron 105 casos que cumplieron los criterios de inclusión. De estas, $54(51,4 \%)$ fueron atendidas en el Hospital de San José y 51 (48,6\%) en el Hospital infantil Universitario de San José. La edad promedio en las pacientes fue 48,7 años (DE 13,9), con una mínima de 15 años y una máxima de 85 años. Otras características demográficas se ilustran en la Tabla 1.

De las 105 pacientes $97(92,4 \%)$ tuvieron una ecografía mamaria, de las cuales $60(61,9 \%)$ fueron con intención diagnóstica y 24 pacientes $(38,1 \%)$ como

\begin{tabular}{|l|c|c|}
\hline \multicolumn{3}{|c|}{ Tabla I. Características de la población } \\
\hline Hospital de San José & n= 105 & $\%$ \\
\hline Hospital Infantil Universitario de San José & 54 & $(51.4)$ \\
\hline Edad, promedio (DE), años & 48,7 & $(48.6)$ \\
\hline Nuliparidad & 20 & $(13.9)$ \\
\hline Menarquia, promedio (DE), años* & 13,3 & $(1.5)$ \\
\hline Menopausia † & 51 & $(48.6)$ \\
\hline edad menopausia, promedio (DE), años & 48,7 & $(4.5)$ \\
\hline Terapia reemplazo hormonal & 6 & $(5.7)$ \\
\hline Antecedente familiar cáncer de mama $\S$ & 13 & $(12.4)$ \\
\hline Antecedente personal cáncer de mama & 2 & $(1.9)$ \\
\hline Motivo de consulta & & \\
\hline Hallazgo imaginológico & 49 & $(46.7)$ \\
\hline Masa & 29 & $(27.6)$ \\
\hline Dolor & 19 & $(18.1)$ \\
\hline Otro & 8 & $(7.6)$ \\
\hline
\end{tabular}

*Menarquía temprana:primer periodo menstrual antes de los 12 años de edad (23); †menopausia: años cumplidos al momento de la última menstruación; ¥ uso de terapia de reemplazo posterior a la menopausia de forma continua; §antecedente familiar de cáncer de mama: materno, paterno o hasta segundo grado de consanguinidad. complemento a la mamografía. Del total de mujeres con estudio ecográfico de la mama, 72 tuvieron un reporte de BI-RADS 4A.El hallazgo ecográfico más frecuente en este grupo fue nódulo en 52 (72,2\%), seguido por quiste complejo en doce $(16,7 \%)$ y el complicado en tres pacientes $(4,2 \%)$. Veinticinco pacientes tuvieron un examen físico normal y 22 se presentaron con una masa palpable.

Del total, $88(83,8 \%)$ tuvieron una mamografía como parte de su estudio, 39 de ellas $(44,3 \%)$ con una indicación de tamización y $49(55,7 \%)$ con intención diagnóstica. De las 88 mujeres con mamografías, 36 tuvieron un reporte BI-RADS 4A. Dentro de este subgrupo, 19 mujeres fueron con intención de tamización y 17 con indicación diagnóstica. Veinticinco tuvieron un examen clínico de la mama negativo. La evaluación de la distribución de los hallazgos mamográficos en el subgrupo de BI-RADS 4A mostró que lo más frecuente fueron las microcalcificaciones en 23 casos $(63,9 \%)$, seguido por nódulos en doce $(33,3 \%)$ y asimetría en cuatro $(11,1 \%)$.

Solo tres pacientes tuvieron un hallazgo de BI-RADS 4A simultáneo tanto en eco mamaria como en mamografía, observando en esta última dos nódulos y una con microcalcificaciones y en la ecografía fueron dos quistes complejos y un nódulo. En los tres casos al examen clínico de la mama era negativo. La mayoría de las lesiones por mamografía y ecografía mamaria se encontraron en los cuadrantes superoexternos de ambas mamas como se ilustra en las Figuras 1 y 2 . Todas las pacientes clasificadas como BI-RADS 4A, tanto por mamografía o por ecografía mamaria fueron sometidas a estudio patológico, fue necesario en algunos casos más de una biopsia para estudio conclusivo. Las técnicas utilizadas para el estudio patológico se reportan en la Tabla 2. La tasa de complicaciones relacionadas con el procedimiento fue $2,85 \%$ (tres pacientes), registradas como dos hematomas en el sitio de la toma y una infección.

En siete pacientes el diagnóstico fue cáncer de mama, como se reporta en la Tabla 3, lo cual da un valor predictivo positivo (VPP) de 6,7\%. Cuatro de 72 casos con BI-RADS 4A por ecografía tuvieron diagnóstico de cáncer de mama (VPP de 5,5\%) mediante biopsia con 


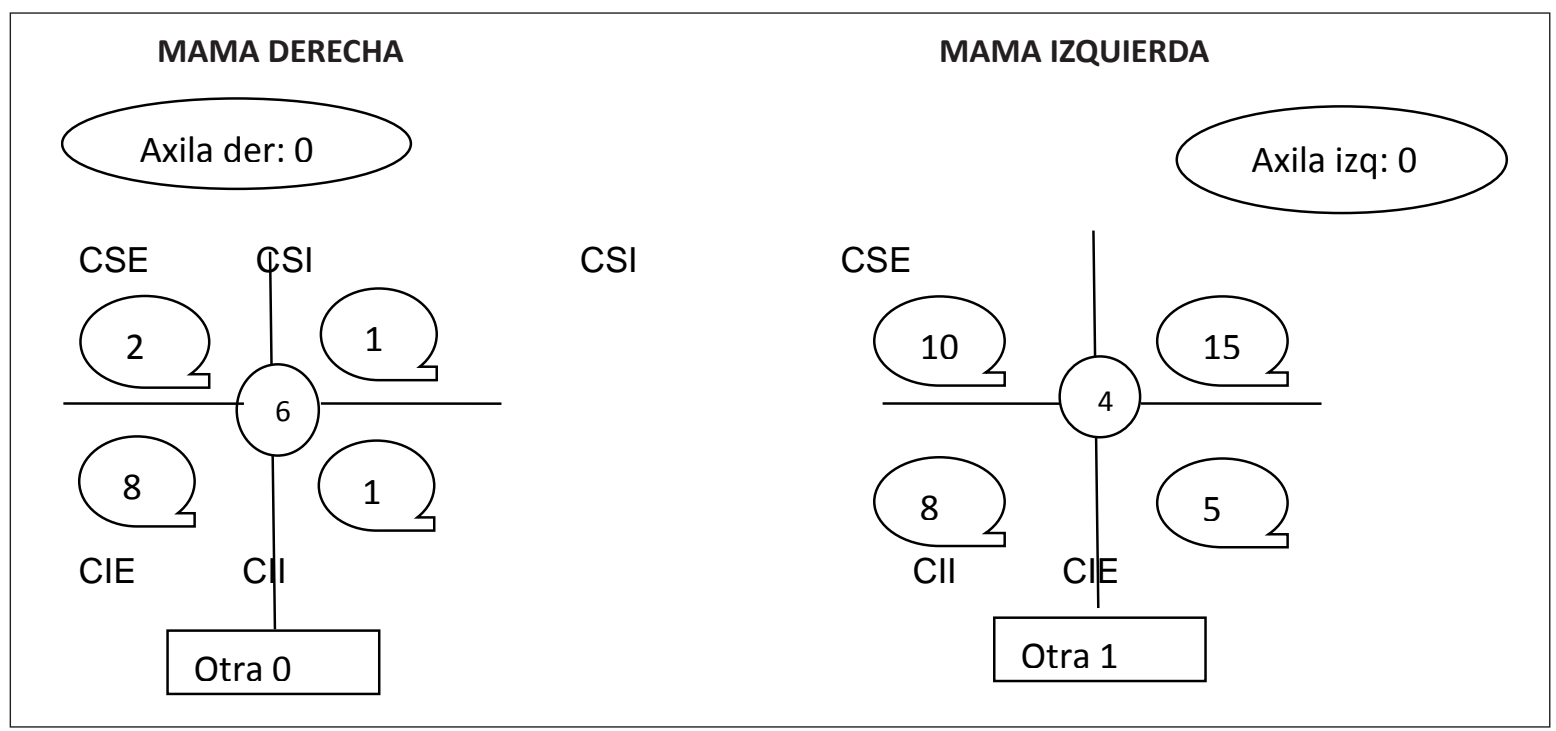

Figura I. Localización de lesiones en mamografía BI-RADS 4A. Una paciente puede tener más de una lesión. Se describen frecuencias absolutas. CSE: cuadrante supero-externo, CSI: cuadrante supero-interno, CIE: cuadrante ínfero-externo, Cll: cuadrante ínfero-interno

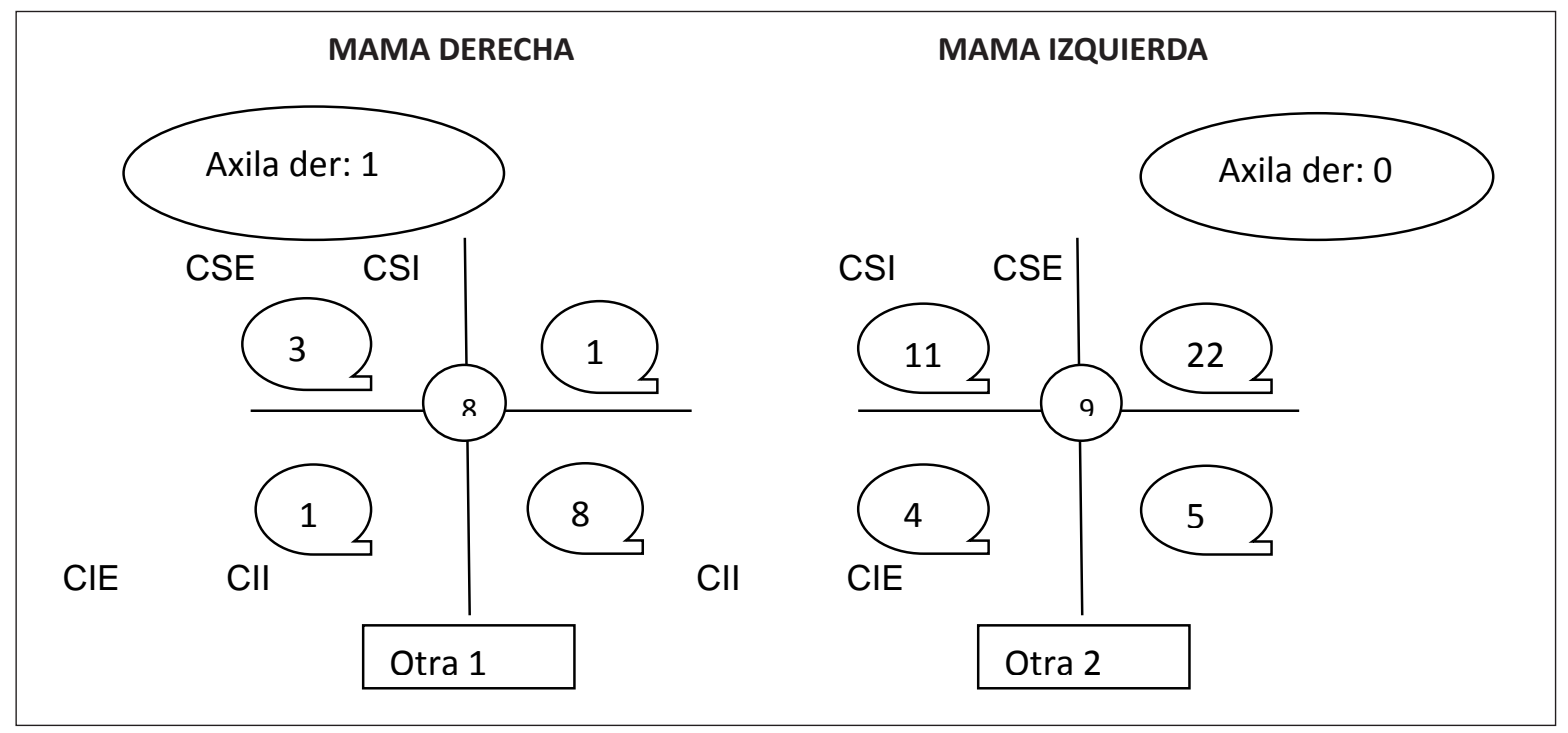

Figura 2. Localización de lesiones en ecografía BI-RADS 4A. Una paciente puede tener más de una lesión. Se describen frecuencias absolutas.CSE: cuadrante supero-externo, CSI: cuadrante supero-interno, CIE: cuadrante ínfero-externo, Cll: cuadrante ínfero-interno.

aguja trucut y todos fueron nódulos. No se encontró malignidad en los quistes complejos o complicados. Tres de 36 pacientes con BI-RADS 4A mamográfico tuvieron diagnóstico de cáncer (VPP de 8,3\%). Los tres casos presentaron microcalcificaciones, sin masa palpable.
Dos fueron evaluadas con biopsia abierta guiada con arpón y uno con trucut, pero no por estereotaxia. Ninguno de los tres casos con BI-RADS 4A simultaneo en ecografía y mamografía tuvieron diagnóstico de cáncer. Las características patológicas de las pacientes con diagnóstico de cáncer se reportan en la Tabla 4. 


\begin{tabular}{|l|c|c|}
\hline \multicolumn{3}{|c|}{ Tabla 2. Tipo y número de biopsias } \\
\hline ACAF* & $\mathbf{n}$ & $\%$ \\
\hline Trucut $\dagger$ & 33 & $(31.4)$ \\
\hline Estereotaxia & 64 & $(60.9)$ \\
\hline Mamo tome & 22 & $(20.9)$ \\
\hline Arpón & 2 & $(1.9)$ \\
\hline Incisional & 12 & $(11.4)$ \\
\hline Escisional & I & $(0.95)$ \\
\hline Numero de biopsias & 24 & $(22.9)$ \\
\hline una & & \\
\hline más de una & 58 & $(55.2)$ \\
\hline sin dato & 46 & $(43.8)$ \\
\hline
\end{tabular}

*Aspiración con aguja fina; † biopsia cortante.

\section{Discusión}

La evaluación por ultrasonografía de la mama ha demostrado su utilidad no solo para diferenciar entre lesiones quísticas y sólidas, sino también benignas y malignas. En su clásico estudio Stavros y col. proponen una serie de descriptores que pudieron diferenciar en forma confiable masas malignas de benignas con 98,4\% de sensibilidad y $99,5 \%$ de valor predictivo negativo. ${ }^{7}$ Este reporte fue quizá el primer intento de organizar de manera sistemática las descripciones de los hallazgos ecográficos de tal forma que sirvieran para predecir la posibilidad de malignidad.

Para el año 2003 la cuarta edición del BI-RADS incluyó un lexicón propio para el reporte ecográfico que incluyó descriptores específicos como forma de la masa, orientación, márgenes, características acústicas posteriores, vascularización y un ítem conocido como "otras características". ${ }^{8}$ Basado en la combinación de estos descriptores, el radiólogo puede categorizar la lesión de acuerdo con la probabilidad de malignidad.

La nomenclatura BI-RADS 4 es asignada a casos sospechosos para las cuales se recomienda un estudio histopatológico. ${ }^{4}$ Sin embargo se asocia con amplia variabilidad en el pronóstico y con una probabilidad de malignidad entre $2 \%$ y $95 \% .{ }^{9}$ Por esta razón, a partir de la cuarta edición la clase 4 ha sido dividida en 3 subcategorías (4A, 4B, 4C). La BI-RADS 4A tiene

\begin{tabular}{|l|c|c|}
\hline \multicolumn{3}{|c|}{ Tabla 3. Reporte de patología } \\
\hline Benigna & $\mathbf{n}$ & $\%$ \\
\hline Hiperplasia sin atipias & 86 & $(82.7)$ \\
\hline Hiperplasia proliferativa con atipia & 6 & $(5.8)$ \\
\hline Carcinoma ductal in situ & 5 & $(4.8)$ \\
\hline Carcinoma ductal infiltrante & 2 & $(1.9)$ \\
\hline Otros tipos histológicos & 4 & $(3.8)$ \\
\hline
\end{tabular}

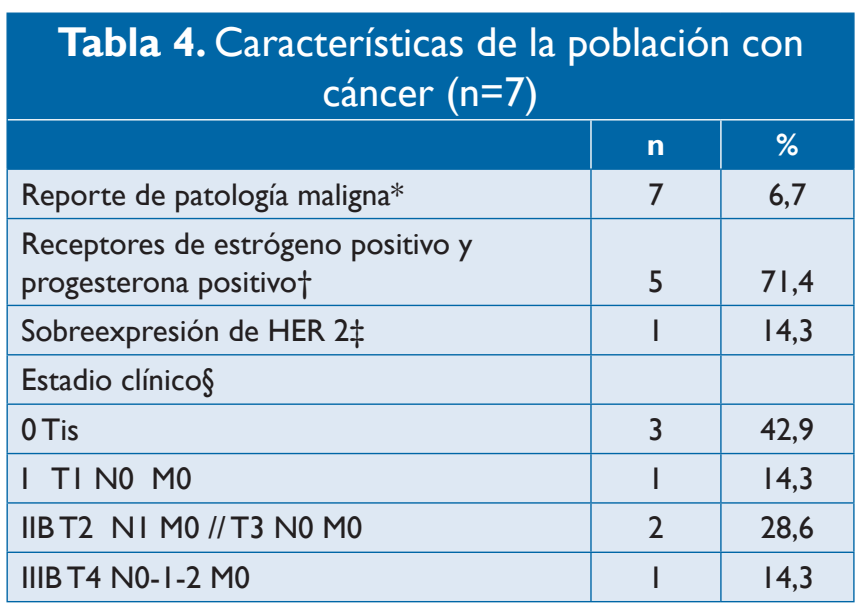

*Pacientes con diagnóstico de cáncer mediante reporte histopatológico; $\dagger$ †atos disponibles para cinco pacientes; łdatos disponibles para tres pacientes; $\S$ clasificación por estadios del cáncer de mama, dependiendo de la valoración histopatológica del TNM.

la más baja probabilidad de malignidad de las tres, estimada entre 2 y $10 \% .^{4}$

A pesar de la recomendación de realizar un estudio patológico para todas las sub-categorías del BI-RADS 4, esta sirve para brindar una mejor información al comunicar un nivel de sospecha más específico al médico y al paciente ${ }^{8}$, así como para prepararlos sobre los resultados de la biopsia. ${ }^{10}$ Estas subcategorías también pueden ser utilizadas para llevar a cabo una auditoría interna, evaluar la correlación radiológica-patológica y mejorar la investigación dirigida a imágenes. ${ }^{4}$

La mayoría de los diagnósticos definidos en esta categoría son masas sólidas con características de benignidad, algunas de ellas palpables, quistes complicados y algunos quistes complejos o masas sólidas sugestivas de abscesos. ${ }^{4}$ De hecho, el hallazgo ecográfico más frecuente en este grupo fue el nódulo en 72,2\% de los casos, seguido por el quiste complejo $(16,7 \%)$ y el quiste complicado $(4,2 \%)$. 
En general puede decirse que en la ecografía las masas sólidas de forma redondeada o elipsoide, con menos de tres lobulaciones, de márgenes circunscritos, sin evidencia de hiperecogenicidad o hipoecogenicidad, sin sombra acústica posterior ni micro calcificaciones internas y paralela, son probablemente benignas. La orientación, la forma y las márgenes parecen ser las características más importantes para diferenciar lesiones malignas de benignas. ${ }^{11}$

Los quistes complicados pueden tener ecos internos de bajo nivel, focos ecogénicos o septos delgados en su interior. ${ }^{12}$ Dado que menos del $1 \%$ de los quistes complicados pueden ser cáncer ${ }^{13}$, estos deberían clasificarse como lesiones probablemente benignas según los criterios de BI-RADS y se manejarían con seguimiento a seis meses y no requerir siempre una biopsia. Los quistes complejos suelen tener pared y septos gruesos (mayores de $0,5 \mathrm{~mm}$ ) o con un componente sólido asociado en su interior. ${ }^{12}$ En estas circunstancias la probabilidad de malignidad puede ser hasta de $30 \%{ }^{14}$ lo cual ratifica su condición BIRADS 4, aunque puede ser mejor clasificado dentro del subgrupo de BIRADS $4 b$.

La recomendación para las subcategorías del BIRADS 4 es el estudio patológico. ${ }^{5}$ Todas las pacientes clasificadas como BI-RADS 4A en nuestro estudio fueron sometidas a una evaluación patológica. Las técnicas utilizadas se reportan en la Tabla 2. Las biopsias abiertas escisionales han sido desplazadas por técnicas diagnósticas preoperatorias tales como la aspiración con aguja fina y las biopsias con aguja cortante que toman un núcleo de tejido conocidas comúnmente como agujas trucut. Estas biopsias guiadas por ecografía mamaria ofrecen una visualización directa de la aguja durante la toma lo cual permite una mejor muestra del tejido. ${ }^{15}$ Por lo regular se utiliza una aguja trucut calibre 14 o mayor con la cual se obtiene un volumen de tejido adecuado para diagnóstico patológico. Sin embargo, se han empezado a utilizar dispositivos de biopsia asistidos por vacío los cuales pueden obtener mayores volúmenes de tejido lo cual reduce la eventualidad de falsos negativos en el reporte patológico. ${ }^{16}$
La presente revisión muestra un valor predictivo positivo de $5,5 \%$ que se encuentra dentro del rango descrito en el BI-RADS. Lazarus y col. encontraron una frecuencia de cáncer del $6 \%$ en la subcategoría $4 \mathrm{~A} .{ }^{10} \mathrm{Sin}$ embargo, otros estudios han informado resultados patológicos malignos más altos para el BIRADS $4^{\mathrm{a}}$, desde $7,6 \%$ hasta $41 \% .8,9$

El dato de 5,5\% puede reflejar mayor libertad para esta subcategoría, pero también cierto grado de inseguridad, marcando lesiones para biopsia que a lo mejor se clasifican como BI-RADS 3 y ameritan solo un seguimiento a corto plazo. Es claro que el miedo de pasar por alto el cáncer es un potente impulsor para el exceso de biopsias. ${ }^{17}$

A pesar de la guía definida en el lexicón, no hay criterios estrictos para designar una determinada lesión como 4A, por tanto esa decisión puede estar influida por criterios subjetivos del radiólogo como muchos estudios lo han reportado. Se ha demostrado un nivel de acuerdo moderado para la forma de la masa, orientación, límites y característica acústica posterior. ${ }^{1,4,8,10}$, Sin embargo, existe una menor concordancia para la descripción del patrón de ecogenicidad, los márgenes de la lesión y las características acústicas posteriores. ${ }^{1,4,10}$, Los valores predictivos más importantes para diferenciar una lesión benigna de una maligna son las márgenes espiculadas, la forma irregular y la orientación antiparalela a la piel que constituyen un alto valor predictivo de malignidad. ${ }^{18}$

Se ha encontrado un nivel moderado de acuerdo para la categorización definitiva del BI-RADS 4 y 5, pero un pobre nivel de acuerdo cuando se incluyen las subcategorías 4A, 4B y 4C.110 Parte de esta variabilidad puede ser consecuencia de la técnica del operador, el nivel de entrenamiento, la experiencia del radiólogo o de confusión al usar el lexicón para la ultrasonografía. ${ }^{4}$

Dado el sustancial número de biopsias realizadas para lesiones benignas, se han hecho intentos para identificar nuevos umbrales que redefinan las recomendaciones de biopsia, teniendo en cuenta que los procedimientos no son inocuos y se asocian con complicaciones como hematomas, infección, cicatrices, dolor, ansiedad y miedo 
al diagnóstico, sin contar los gastos financieros. ${ }^{17} \mathrm{La}$ estandarización de reglas simples y exactas para clasificar una masa dentro de la categoría 4A, 4B o 4C puede ayudar a disminuir la variabilidad del reporte y definir mejor la indicación del estudio patológico. Flowers y col. publicaron un estudio piloto en el cual el radiólogo asignaba estimados de riesgo medido en porcentaje de probabilidad para carcinoma ductal in situ y carcinoma infiltrante a pacientes con categoría BI-RADS 4 y 5 de acuerdo con parámetros estrictamente definidos. Se encontró que si se someten a biopsia solo aquellas pacientes con riesgo de carcinoma in situ o infiltrante mayor del $10 \%$, evitaban el $22 \%$ de los procedimientos y no se incurría en demoras en el diagnóstico de alguna lesión maligna, logrando una tasa de biopsias positivas de $36 \%$ para la subcategoría $4 \mathrm{~A} .{ }^{17}$ En otro estudio Menezes y col. evaluaron la variabilidad interobservador en una institución usando definiciones muy claras para la categorización. Encontraron un mejor nivel de acuerdo en la subcategorización del BI-RADS 4 y lograron un valor predictivo positivo de malignidad del $17 \%$ para el BI-RADS 4A. Estos criterios son fáciles de implementar en cualquier servicio de imágenes. ${ }^{9}$

Dado el bajo número de casos clasificados como BIRADS 4A mamográfico en este estudio, resulta difícil sacar conclusiones válidas. De hecho, el VPP en esta cohorte resultó ser muy inferior al valor encontrado en el primer estudio ${ }^{6}$ el cual fue del $12 \%$. Con alta probabilidad, el menor tamaño de la muestra pudo influir en estos resultados.

Existe sin embargo evidencia que igual que en los reportes ecográficos, también los hallazgos mamográficos son susceptibles de sufrir interpretaciones diferentes de acuerdo con el radiólogo. En el estudio de Lazarus y col. para la mamografía hubo un nivel de acuerdo moderado para la forma de la masa y los márgenes, pero pobre acuerdo en relación con la densidad. En cuanto a las calcificaciones en general hubo poco acuerdo con respecto a sus características así como en la evaluación de la distorsión de la arquitectura. ${ }^{10}$

Los resultados de este estudio son un intento por condensar la información heterogénea proveniente de reportes de estudios de imágenes de la mama de diferentes centros radiológicos, teniendo en cuenta que las dos instituciones participantes son centro de referencia para enfermedades mamarias de varias empresas prestadoras de salud de Bogotá y algunas ciudades vecinas. Sus resultados no siempre pueden aplicarse a centros de atención integral en patología mamaria, en donde un mismo equipo de radiólogos con suficiente experticia en la lectura de imágenes mamarias es el encargado del reporte imaginológico. Muchos de estos estudios pudieron ser catalogados como BI-RADS 3 por parte de los radiólogos.

\section{Referencias}

1. Abdullah N, Mesurolle B, El-Khoury M, Kao E. Breast imaging reporting and data system lexicon for US: interobserver agreement for assessment of breast masses. Radiology. 2009 sep; 252(3): 665-72.

2. American College of Radiology. Breast Imaging Reporting and Data System. 2nd ed. Reston, VA: American College of Radiology; 1995.

3. Kerlikowske K, Grady D, Barclay J, Frankel SD, Ominsky SH, Sickles EA, et al. Variability and accuracy in mammographic rpretation using the American College of Radiology Breast Imaging Reporting and Data System. J Natl Cancer Inst. 1998 Dec 2; 90(23):1801-9.

4. Raza S, Goldkamp AL, Chikarmane SA, Birdwell RL. US of breast masses categorized as BI-RADS 3, 4, and 5: pictorial review of factors influencing clinical management. Radiographics. 2010 Sep; 30(5):1199-213.

5. Garcia Angulo OA, Guio Avila JI. Frecuencia de patología mamaria maligna en pacientes con mamografia BIRADS 4A. Rev Colomb Radiol. 2011 Dic; 22(4): 3352-6.

6. Stavros AT, Thickman D, Rapp CL, Dennis MA, Parker SH, Sisney GA. Solid breast nodules: use of sonography to distinguish between benign and malignant lesions. Radiology. 1995 Jul; 196(1):123-34.

7. Lee HJ, Kim EK, Kim MJ, Youk JH, Lee JY, Kang DR. Observer variability of Breast Imaging Reporting and Data System (BI-RADS) for breast ultrasound. Eur J Radiol. 2008 Feb; 65(2):293-8.

8. Jales RM, Sarian LO, Torresan R, Marussi EF, Alvares BR, Derchain S. Simple rules for ultrasonographic subcategorization of BI-RADS - US 4 breast masses. Eur J Radiol. 2013 Aug; 82(8):1231-5.

9. Lazarus E, Mainiero MB, Schepps B, Koelliker SL, Livingston LS. BI-RADS lexicón for US and mammography: interobserver variability and positive predictive value. Radiology. 2006 May; 239(2):385-91.

10. Chala L, Endo E, Kim S, de Castro F, Moraes P, Cerri G, et al. Gray-scale sonography of solid breast masses: diagnosis of probably benign masses and reduction of the number of biopsies. J Clin Ultrasound. 2007 Jan; 35(1):9-19.

11. Rinaldi P, Ierardi C, Costantini M, Magno S, Giuliani M, Belli P, et al. Cystic breast lesions. Sonographic findings and clinical management. J Ultrasound Med. 2010 Nov; 29(11):1617-26.

12. Venta LA, Kim JP, Pelloski CE, Morrow M. Management of complex breast cysts. AJR Am J Roentgenol. 1999 Nov; 173(5):1331-6.

13. Berg WA, Campassi CI, Ioffe OB. Cystic lesions of the breast: sonographic pathologic correlation. Radiology. Apr; 227(1):183-91.

14. American College of Radiology (2003). Breast Imaging Reporting and Data System ${ }^{\circledR}\left(\right.$ BI-RADS $\left.{ }^{\circledR}\right)$ Atlas.. 5th ed.: Reston, VA.; (2003).

15. Perrot N, Jalaguier-Coudray A, Frey I, Thomassin-Naggara I, Chopier J. USguided core needle biopsy: false-negatives. How to reduce them?. Eur J Radiol. 2013 Mar; 82(3):424-6.

16. Schueller G SWCHT. Accuracy of ultrasound-guided, large-core needle breast biopsy. Eur Radio. 2008 Sep; 18(9):1761-73.

17. Flowers CI, O'Donoghue C, Moore D, Goss A, Kim D, Kim JH, et al. Reducing false-positive biopsies: a pilot study to reduce benign biopsy rates for BI-RADS 4A/B assessments through testing Risk stratification and new thresholds for intervention. Breast Cancer Res Treat. 2013 Jun;139(3):769-77.

18. Hong AS, Rosen EL, Soo MS, Baker JA. BI-RADS for sonography: positive and negative predictive values of sonographic features. AJR Am J Roentgenol. 2005 Apr; 184(4):1260-5. 